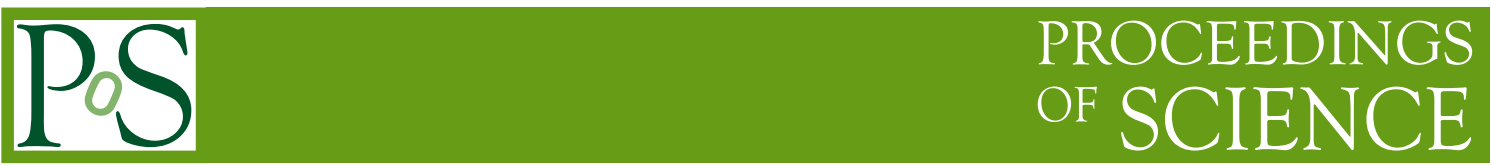

\title{
Physics with $\tau$ Lepton Final States in ATLAS
}

\author{
Nils Ruthmann* on behalf of the ATLAS Collaboration \\ Institut für Physik, Universität Freiburg \\ E-mail: ruthmann@cern.ch
}

Heading into its third year of data-taking, ATLAS has already published several of measurements and searches for processes with $\tau$ leptons in the final state. In this note a broad overview over the ATLAS physics programme involving $\tau$ leptons is given. Searches for a SM Higgs boson, charged Higgs bosons as proposed in supersymmetric models and searches for supersymmetry in events with tau leptons are presented as well as Standard Model measurements. The first measurement of the $\tau$ polarization in $W \rightarrow \tau \nu$ decays at a hadron collider and the challenging measurement of the $t \bar{t}$ cross-section in the $\tau_{h} l$ final state are discussed. All observations agree well with the SM prediction.

The XIth International Conference on Heavy Quarks and Leptons,

June 11-15, 2012

Prague, Czech Republic

* Speaker. 


\section{Introduction}

The fermionic particle content of the Standard Model (SM) consists of three generations of quarks and leptons. Within those, the tau is the heaviest charged lepton $\left(m_{\tau}=1.77 \mathrm{GeV} / \mathrm{c}^{2}\right)$ and is rather short-lived $(c \tau \approx 87 \mu \mathrm{m})$. It decays via weak interactions dominantly into one or more charged hadrons and one neutrino $(B R \approx 65 \%)$, or leptonically into a light lepton and a pair of neutrinos. A crucial part of the SM is the breaking mechanism of the electroweak symmetry, the Higgs mechanism, which adds one neutral scalar particle to the SM particle content. The mass of this particle is a free parameter of the SM and is only weakly constrained by electroweak precision data. Therefore direct searches are of major interest and were performed at LEP and the Tevatron and are a keypoint in the ATLAS physics programme.

The Large Hadron Collider (LHC) is a proton-proton collider taking collision data since 2009 at center of mass energies between $900 \mathrm{MeV}$ and $8 \mathrm{TeV}$. In 2011 the LHC delivered $4.7 \mathrm{fb}^{-1}$ of integrated luminosity of high quality collision data to the experiments allowing precise measurements of known SM processes as well as good progress in the search for yet unknown phenomena. The ATLAS Experiment [1] at the LHC is a multi-purpose detector build not only to discover or exclude the existence of the Higgs boson, but to maximise the discovery potential of physics beyond the SM in general. The high granularity of the electromagnetic and hadronic calorimeters of the ATLAS detector allow a precise identification and energy measurement of hadronically decaying tau leptons $\left(\tau_{h}\right)$.

Within the ATLAS physics programme $\tau$ leptons play a crucial role in the search for the Higgs bosons, searches for supersymmetric extensions of the SM or measurements of SM processes. In the search for the Higgs boson the, $\tau$ lepton probes the leptonic Higgs-Yukawa coupling which is not accessible from decays to a pair of photons or heavy bosons $(W W / Z Z)$. In many proposed supersymmetric models $\tau$ leptons are of special interest due to enhanced couplings and final state signatures with $\tau$ leptons. Such decays are distinguishable from QCD jets by their narrow shower profile and low track multiplicity, while leptonic tau decays can only be distinguished from prompt electrons or muons using additional information about the event, e.g. the presence of large missing transverse energy or additional leptons of a different flavor.

\section{Search for the SM Higgs boson decaying in a pair of $\tau$ Leptons}

The Higgs boson is produced in LHC collisions mainly via gluon fusion, vector boson fusion or Higgs-Strahlung processes. The Higgs boson branching ratios depend heavily on its mass. At masses below the kinematical threshold for decays into a pair of $W$ or $Z$ bosons, decays to a pair of $b$ or $c$ quarks, a pair of $\tau$ leptons or a pair of gluons are statistically dominant. Due to the large hadronic background in hadron collisions the search in quark and gluon final states are extremely challenging. The decay into $\tau \tau$ accounts for roughly $5-10 \%$ of all Higgs decays for $m_{H}<130$ $\mathrm{GeV}$. Each of the $\tau$ leptons can decay leptonically or hadronically leading to either full hadronic, semi-leptonic or leptonic final states. A search for the SM Higgs boson decaying into a pair of $\tau$ leptons was performed on $\int \mathscr{L} d t=4.7 \mathrm{fb}^{-1}$ collision data recorded in 2011 in each of these three sub-channels [2]. 


\subsection{Irreducible $Z \rightarrow \tau \tau$ background}

For all $\tau$ decay modes the largest background source are irreducible $Z \rightarrow \tau \tau$ decays leading to a signal like event topology. The main discriminating variable is the invariant ditau mass. Its correct modeling is therefore of great importance in this search. Due to lepton universality the kinematic properties of $Z \rightarrow l l$ decays are independent of the lepton flavour. Since the coupling of a SM Higgs boson to muons is negligibly small, $Z \rightarrow \mu \mu$ events offer a signal free control sample of $Z$ decays. The muons in these events are removed and replaced by simulated $\tau$ decays. These embedded $Z \rightarrow \tau \tau$ events offer a data-driven estimate of the $Z$ event kinematics along with a fully data-driven description of underlying event and multiple proton-proton interaction activity. Systematic uncertainties account for potential biases from the $Z \rightarrow \mu \mu$ selection criteria.

\section{2 $H \rightarrow \tau_{h} \tau_{h}$}

With both $\tau$ leptons decaying hadronically, this sub-channel suffers most from multijet background. Events are selected with a ditau trigger. Two oppositely charged $\tau_{h}$ candidates and exactly zero light leptons are required. The ditau mass is estimated in the collinear approximation - assuming that the neutrinos from the $\tau$ decays are collinear to the visible decay products. The sub-channel focuses on the analysis of $\mathrm{H}+1$ jet events, requiring at least one jet with transverse momentum $p_{T}>40 \mathrm{GeV}$ and missing transverse energy of $E_{T}^{\text {miss }}>20 \mathrm{GeV}$. The selection favors boosted Higgs decays by requiring a small opening angle $\Delta R(\tau, \tau)<2.2$, and a large invariant mass of the $\mathrm{H}+$ jet system $m_{j \tau \tau}>225 \mathrm{GeV}$. This effectively suppresses multijet and $Z \rightarrow \tau \tau$ background. The $Z \rightarrow \tau \tau$ and multijet backgrounds are estimated by fitting the two-dimensional track multiplicity spectrum of both hadronic tau candidates. The multijet template is extracted from data using same sign events, while the template for the $Z \rightarrow \tau \tau$ background is extracted from simulation. Performing the same fit in the signal region yields the background contribution from multijet events. Other backgrounds are estimated by simulation and are subtracted in the fitting method. Systematic uncertainties on these background estimations are $11.6 \%$ for the $Z \rightarrow \tau \tau$ and $22 \%$ for the multijet background respectively. Figure 1 shows the collinear mass spectrum obtained in the control region as well as in the signal region.

\section{$2.3 H \rightarrow \tau_{l} \tau_{h}$}

The semi-leptonic channel benefits from the presence of exactly one light lepton which is used to trigger the event and exactly one $\tau_{h}$. This signature is easily faked by $W+$ jets events which is the second most important background process in this sub-channel. Events are selected by a single lepton $(\mathrm{e} / \mu)$ trigger and are required to have no additional light leptons and exactly one oppositely charged $\tau_{h}$ candidate. To suppress $W+$ jets contributions the transverse mass of the lepton and the missing transverse energy is required to be $m_{T}\left(l, E_{T}^{\text {miss }}\right)<30 \mathrm{GeV}$. Events are categorised based on their event topology in seven analysis categories. These include zero jet events with low and high missing transverse energy and events with at least one jet, each separated by lepton flavour. In addition one category focuses on events with two energetic jets at large pseudo-rapdities in both hemispheres of the detector, as expected in vector boson fusion events. Background from misidentified $\tau_{h}$ candidates are estimated from data using same sign events. For processes in which the jet faking the $\tau_{h}$ signal exhibits a charge correlation to the light lepton (e.g. $W+$ jets) more 


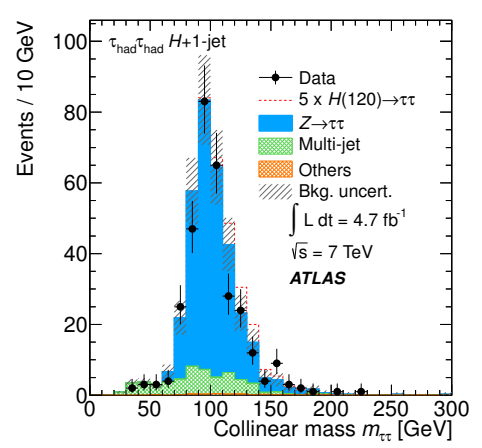

(a)

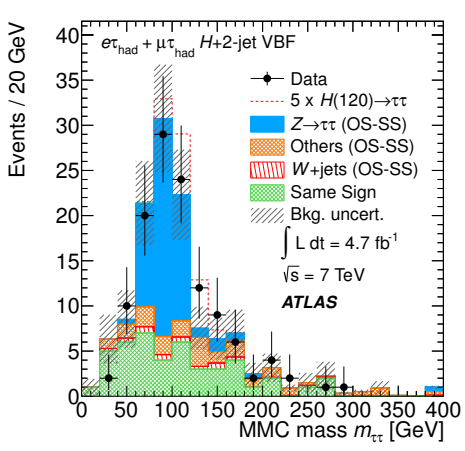

(b)

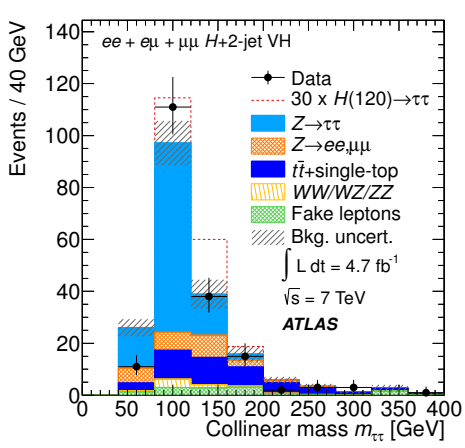

(c)

Figure 1: Ditau mass distributions in the $\tau_{h} \tau_{h}+1$ Jet- (a), the $\tau_{l} \tau_{h}$ VBF- (b) and the $\tau_{l} \tau_{l}$ VH-category.[2]

opposite sign events might be expected. The additional contribution from $W+$ jets events is therefore estimated from a control region at high transverse mass. For other backgrounds, like top-pair production, the additional contribution on top of the same sign yield is estimated from simulation. The ditau mass is fully reconstructed by fitting the $v$ four-momenta in the allowed phase-space. Figure 1 (b) shows the invariant ditau mass spectrum for the Vector Boson Fusion (VBF) category.

\section{$2.4 H \rightarrow \tau_{l} \tau_{l}$}

A combination of single and dilepton triggers is used to trigger events with two isolated leptons for the full leptonic sub-channel. To reduce the large $Z \rightarrow l l$ background the invariant dilepton mass is required to be within $30 \mathrm{GeV}<m_{l l}<100 \mathrm{GeV}$ in the $e / m u$ channel and within $30 \mathrm{GeV}<m_{l l}<75$ $\mathrm{GeV}$ in dielectron and dimuon final states. Events are analysed in four categories, being a vector boson fusion category, a $\mathrm{H}+2$ jets, $\mathrm{H}+1$ jet and $\mathrm{H}+0$ jets category. The $\mathrm{H}+2$ jets category is selecting event topologies as favoured by Higgs boson production in association with a heavy vector boson. In contrast to the VBF process this event topology favours small opening angles between the two jets. To suppress $t \bar{t}$ background, events are rejected if one jet is identified as originating from a $b$-quark. The remaining $Z \rightarrow l l$ background contribution is estimated in low and high $E_{T}^{\text {miss }}$ control regions. The contamination from events with misidentified leptons is estimated by a template fit to the transverse momentum spectrum in a control region with reversed isolation requirements on the leptons. Figure 1 (c) shows the invariant ditau mass spectrum for VH category.

\subsection{Results}

All three sub-channels use a fit to the ditau mass spectrum to set limits on the signal strength parameter $\mu:=\sigma / \sigma_{S M}$ for various Higgs boson mass hypotheses. The result of the combined fit is shown in figure 2.

\section{Search for charged Higgs bosons}

Several theories of physics beyond the SM predict non-minimal electroweak breaking mechanisms. For example the minimal supersymmetric standard model (MSSM) contains two Higgs 


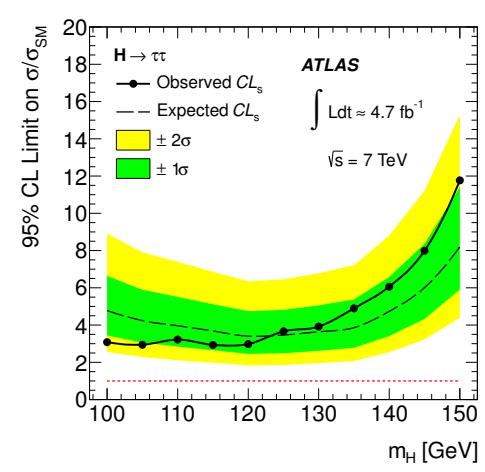

Figure 2: Combined exclusion limit on the Higgs boson production cross-section at 95\% CL from the $H \rightarrow \tau \tau$ analysis.[2]

doublets, resulting in five physical states, two charged Higgs bosons $H^{ \pm}$and three neutral ones $h^{0}, H^{0}, A^{0}$. For small $m_{H^{ \pm}}$, the charged Higgs bosons are predominantly produced via top quark decays. The decay branching ratios depend on the ratio of the vacuum expectation values of the two Higgs doublets $(\tan \beta)$. For values above three, the dominating decay is $H^{ \pm} \rightarrow \tau \nu$. In the following the search for $H^{ \pm}$decaying into $\tau_{h} v$ in $t \bar{t}$ events is described [3]. The final state depends on the decay of the $W^{ \pm}$. Both hadronic and leptonic $W$ decays are considered. Assuming $B R\left(H^{ \pm} \rightarrow \tau v\right)=1$ the search sets upper limits on the branching ratio of the top quark decay $t \rightarrow H^{ \pm} c$.

\section{1 $t \bar{t} \rightarrow b \bar{b} W^{\mp} H^{ \pm} \rightarrow b \bar{b}\left(l^{\mp} v\right)\left(\tau_{h} v\right)$}

The leptonic $W$ decay leads to the presence of an isolated lepton used to trigger the events. Events are required to have no additional light lepton and exactly one $\tau_{h}$ candidate of opposite charge with respect to the light lepton. Furthermore at least two jets are required of which at least one is identified as a $b$-quark initiated jet. To suppress multijet background the sum of transverse momenta of all tracks associated to the primary vertex is required to be $\sum p_{T}>100 \mathrm{GeV}$. Apart from the irreducible background from real $\tau_{h}$, the largest background comes from misidentified $\tau_{h}$ candidates. These contributions are estimated from data by measuring the misidentification probability for electrons in a $Z \rightarrow e e$ control sample and the misidentification probability for jets in a $W+$ jets enriched sample. Systematic uncertainties cover differences introduced by the different jet composition in such a sample compared to the $t \bar{t}$ signal sample. A $H^{ \pm}$mediated $t$ decay would lead to larger missing transverse energy compared to $W$ mediated decays. The $E_{T}^{\text {miss }}$ spectrum in the signal region of the $\tau_{h}+\mu$ channel is shown in figure 3 (a).

\section{$3.2 t \bar{t} \rightarrow b \bar{b} W^{\mp} H^{ \pm} \rightarrow b \bar{b}(q \bar{q})\left(\tau_{h} v\right)$}

In case of a hadronic $W$ decay the final state contains one $\tau_{h}$ and four jets. Therefore a strong suppression of multijet background is needed. Events are selected by a $\tau_{h}+E_{T}^{\text {miss }}$ trigger and are rejected if any light lepton is present. At least four jets are required of which at least one is identified as $b$-quark initiated. Only events with large missing transverse energy $E_{T}^{\text {miss }}>65 \mathrm{GeV}$ are considered which greatly reduces multijet background. Furthermore the event topology must be consistent with the top-quark decay hypothesis, meaning that the invariant mass of one $b$ - and two untagged jets is within a top-quark mass window of $m_{b j j} \in[120,240] \mathrm{GeV}$. The remaining 


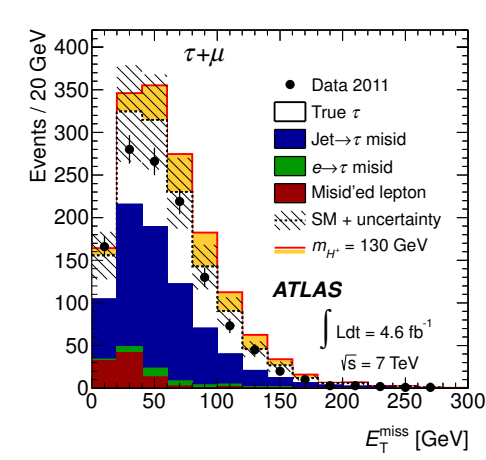

(a)

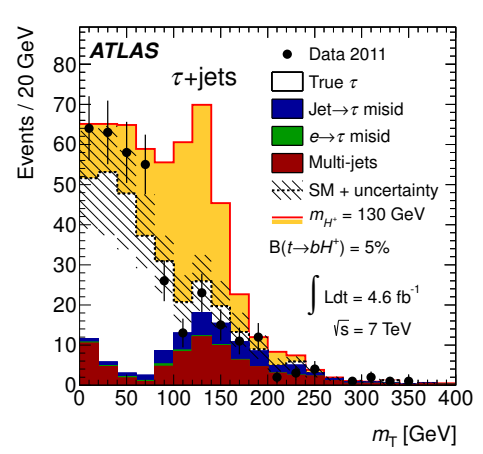

(b)

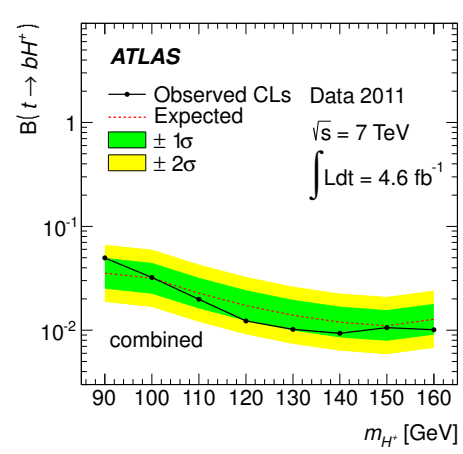

(c)

Figure 3: Final discriminating variables in the $\tau_{h}+\mu$ (a) channel, the $\tau_{h}+$ jets (b) channel and the combined upper limit on $B R\left(t \rightarrow b H^{ \pm}\right)$at $95 \% \mathrm{CL}$ (c). [3]

background from multijet processes is extracted by fitting a template to the missing transverse energy spectrum. The template shapes are taken from control regions with modified identification criteria to enhance the QCD contribution. The transverse mass between the $\tau_{h}$ and $E_{T}^{\text {miss }}$ is used to fit the signal strength parameter and is shown in figure 3 (b).

\subsection{Results}

Apart from the presented analyses with one $\tau_{h}$ candidate also the $\tau_{l}+$ jets channel is considered. All three sub-channels are combined and upper limits on the branching ratio $B R\left(t \rightarrow b H^{ \pm}\right)$ are set for various $m_{H^{ \pm}}$hypotheses. Limits range from $5 \%$ at low masses to $1 \%$ at large $m_{H^{ \pm}}$as shown in figure 3 (c).

\section{Search for supersymmetry in events with $\tau$ lepton final states}

Supersymmetry (SUSY) extends the Poincaré algebra by fermionic generators introducing a symmetry between fermions and bosons. The experimental fact that no supersymmetric particle was observed yet tells that this symmetry needs to be broken if realised in nature. In R-parity conserving models, in which the lightest SUSY particle (LSP) is considered stable, production of SUSY particles would lead to a decay chain down to the next-to-lightest SUSY particle which would decay into its SM partner and the LSP. In large phase-space regions of various SUSY models the SUSY partners of the third generation fermions, e.g. the partner of the $\tau$ lepton usually denoted as $\tilde{\tau}$, are lighter compared to the first or second generation of SUSY particles. Therefore final states with $\tau$ leptons are of particular interest in the search for SUSY with light $\tilde{\tau}$ leptons. ATLAS performed a search for SUSY in events with at least two jets, missing transverse energy and at least one or two $\tau_{h}$ in a datasample of $\int L d t=2.05 \mathrm{fb}^{-1}[4,5]$. These analysis are held inclusively in the considered final state to cover generic SUSY models leading to such event topologies. Since no excess of events is observed an upper limit on the visible cross-section of such phenomena is set and interpreted in the framework of a gauge-mediated SUSY breaking model (GMSB), in which a lower limit on the SUSY breaking scale $\Lambda$ is extracted. Events are selected by a jets + missing transverse energy trigger and required to have at least one (two) $\tau_{h}$ candidates, two jets and 


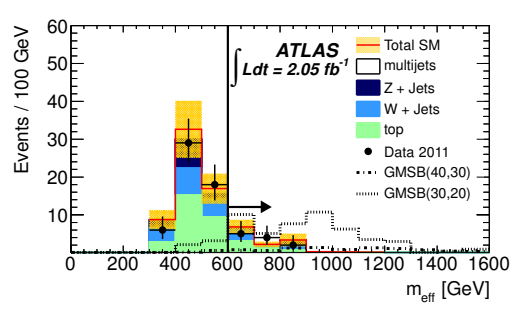

(a)

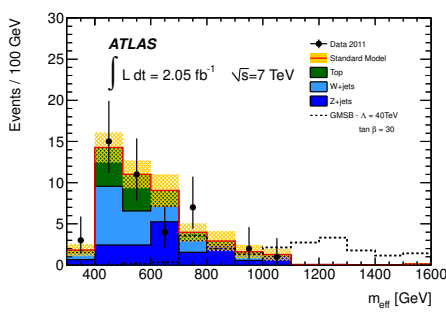

(b)

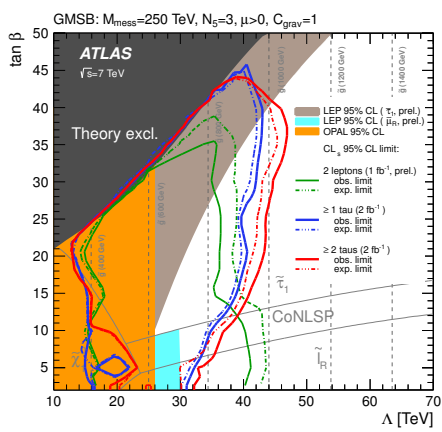

(c)

Figure 4: Effective mass spectra in the $\geq 1 \tau_{h}$ (a) and $\geq 2 \tau_{h}$ (b) analysis as well as the extracted limits on the GMSB model parameters $\tan \beta$ and $\Lambda$. [4, 5]

large missing transverse energy $E_{T}^{\text {miss }}>130 \mathrm{GeV}$. Events with additional electrons or muons are rejected. To reduce contributions from multijet events in which one of the jets is mismeasured, a large angular separation between the jets and the missing transverse energy is required.

\section{1 $\geq 1 \tau_{h}$ analysis}

The dominant background in the inclusive $1 \tau_{h}$ analysis arises from top pair and single top production as well as from $W / Z+$ jets events. Events with small transverse mass $m_{T}\left(\tau_{h}, E_{T}^{\text {miss }}\right)<$ $110 \mathrm{GeV}$ are rejected to suppress $W+$ jets events. A measure of the mass scale $m_{\mathrm{eff}}{ }^{1}$ is computed as the scalar sum of $E_{T}^{\text {miss }}$, the $p_{T}$ of the two leading jets and the $p_{T}$ of the leading $\tau_{h}$. A requirement of $m_{\text {eff }}>600 \mathrm{GeV}$ is effectively suppressing remaining SM processes. The background event yield in the signal region is estimated from dedicated control regions enriched in real $\tau_{h}$ (mainly originating from $\mathrm{W}$ and top-quark decays), in misidentified $\tau_{h}$ from $W / Z+$ jets events and in multijet events. Normalisation corrections are extracted and extrapolated into the signal region using simulation. Systematic uncertainties accounting for imperfections in this extrapolation procedure, e.g. from differences in the event composition, are of the order of 10-20\%. Figure 4 (a) shows the effective mass spectrum in the signal region. With 11 events observed and 13.2 \pm 4.2 expected events the observation is in good agreement with the background only hypothesis.

\section{$4.2 \geq 2 \tau_{h}$ analysis}

In the two $\tau_{h}$ analysis the main backgrounds are $W+$ jets, top-pair and single top production with one real and one misidentified $\tau_{h}$, as well as $Z \rightarrow \tau_{h} \tau_{h}$ decays. Requirements of $m_{\text {eff }}>700$ $\mathrm{GeV}$ and $m_{T, 1}^{\tau_{2}}+m_{T}^{\tau_{1}}>80 \mathrm{GeV}$ suppress most of those SM processes. The remaining contributions are estimated from control regions enriched in $W+$ jets and top-quark events as well as in multijet events respectively. Figure 4 (b) shows the effective mass spectrum in the signal region without the requirement on $m_{\text {eff }}$. Three events are observed while expecting $5.3 \pm 2,6$ events from SM processes.

\footnotetext{
${ }^{1}$ The effective mass $m_{\mathrm{eff}}$ is calculated as the sum of $E_{T}^{\mathrm{miss}}$ and the magnitude of the transverse momenta of the two highest- $p_{T}$ jets and all selected taus.
} 


\subsection{Interpretation of results}

Both analysis set limits on the visible cross-section defined as the product of cross-section, branching-ratio, acceptance and efficiency. The $\geq 1 \tau_{h}$ analysis sets an upper limit of $4 \mathrm{fb}$ while the $\geq 2 \tau_{h}$ analysis sets an upper limit of $2.9 \mathrm{fb}$ on the visible cross-section each at $95 \% \mathrm{CL}$. Furthermore the results are interpreted in the GMSB model which leads to limits in the $\tan \beta-\Lambda$ plane. These are shown in figure 4 (c).

\section{Standard Model measurements}

Beside searches for physics beyond the SM, measurements of known SM processes involving $\tau$ leptons are of major interest in the ATLAS physics programme. These probe the SM at yet unexplored energy scales and directly support BSM searches by measuring rare SM background processes. Two analyses, the measurement of the $t \bar{t} \rightarrow l \tau_{h}+X$ cross-section [6] and the measurement of $\tau$ polarization in $W \rightarrow \tau_{h} v$ decays [7] are presented.

\subsection{Cross-section measurement of the $t \bar{t} \rightarrow l \tau_{h}+X$ process}

Measurements of the $t \bar{t}$ production cross-section are of particular interest as many proposed models of physics beyond the SM predict modified top-quark branching ratios. In the SM top quarks decay in nearly $100 \%$ of all cases weakly into a $b$-quark and a $W$ boson. In this measurement final states with one light lepton $(e / \mu)$, one $\tau_{h}$, at least two jets and missing transverse energy are considered. Events are selected by a single lepton trigger and at least one of the jets is required to be identified as originating from a $b$-quark. A control sample to estimate the background contribution in the signal region is selected by reverting the $b$-tag requirement.

The dominant background comes from $t \bar{t} \rightarrow l+$ jets events where one jet is misidentified as $\tau_{h}$. Therefore the $\tau_{h}$ identification (ID) is crucial to obtain a decent signal purity. Instead of requiring $\tau_{h}$ identification requirements this analysis uses the score spectrum of the $\tau_{h}$ ID boosted decision tree (BDT) [8] to extract the signal contribution in a template fit. The BDT output shape for the background is extracted from the zero $b$-jet control sample after subtracting real $\tau_{h}$ contributions using simulation. Background contributions with misidentified $\tau_{h}$ are estimated by exploiting the missing charge correlation between the lepton and the misidentified $\tau_{h}$ in multijet events, a similar technique as in the SM Higgs boson search outlined above. Events in which the $\tau_{h}$ has the same reconstructed charge as the lepton are subtracted from the opposite sign sample leading to a cancellation of the multijet background. Figure 5 (a) shows the BDT score distribution for real and misidentified $\tau_{h}$ and the event yields of those in the same sign and opposite sign region respectively. A template fit to the $\tau_{h}$ BDT output spectrum in the $b$-tag sample is performed to extract the signal contribution. Figures 5 (b) and (c) show the BDT distributions in the control and signal region together with the fitted signal contribution from single-track hadronic tau decays. Combining $e+\tau_{h}$ and the $\mu+\tau_{h}$ channel yields a measured cross-section of $\sigma_{t \bar{t}}=186 \pm 13$ (stat.) \pm 20 (syst.) \pm 7 (lumi.) pb.

\subsection{Measuring $\tau$ Polarization in $W \rightarrow \tau v$ decays}

Given the short lifetime and their maximal parity violating decays, $\tau$ leptons are the only leptons whose spin information is reconstructable from the decay products recorded in ATLAS. The 


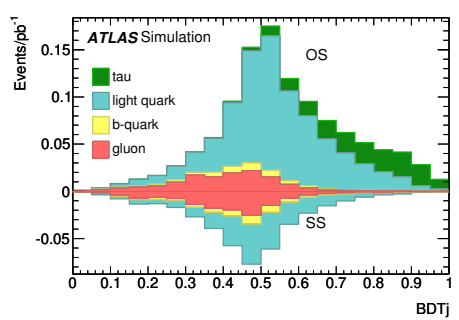

(a)

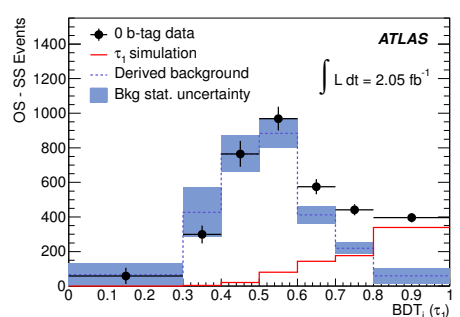

(b)

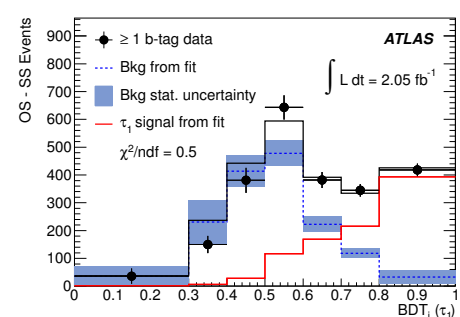

(c)

Figure 5: The $\tau_{h}$-ID BDT score output for signal and background processes (a), the extracted template shapes in the control region (b) together with the fit result in the signal region (c). [6]

$\tau$ polarization $P_{\tau}$ is defined as the asymmetry between the left-handed and right-handed $\tau$ production cross-sections of a given process. In $W \rightarrow \tau v$ decays the SM predicts a polarization of -1 , reflecting the parity violating structure of the charged weak current. Parity conserving interactions like a $H \rightarrow \tau \tau$ decay would yield $P_{\tau}=0$. The analysis therefore is not only the first measurement of $\tau$ polarization at a hadron collider, but also shows ways how to probe coupling structures of yet to be discovered phenomena. The dominant single-track hadronic tau decay mode is $\tau^{-} \rightarrow \rho^{-} v \rightarrow \pi^{-} \pi^{0} v(\mathrm{BR} \approx 25 \%)$. In this decay channel the spin information of the $\tau$ is transferred to the intermediate $\rho$ meson due to the maximal parity violating charged current. Angular momentum conservation results in a preference of left-handed $\tau$ leptons to decay to a transversely polarised $\rho$, leading to a symmetric energy sharing between the two pions in the final state. A longitudinal $\rho$ polarization would be preferred in hypothetical non-SM decays to right-handed tau leptons, leading to an asymmetric energy sharing. An observable constructed to be sensitive to this energy sharing, and therefore the polarization is $\Upsilon$, defined as:

$$
\Upsilon:=\frac{\left(E_{T}^{\pi^{-}}-E_{T}^{\pi^{0}}\right)}{p_{T}^{\tau, \mathrm{vis}}}
$$

The events were recorded in 2010 and selected with a $\tau_{h}+E_{T}^{\text {miss }}$ trigger. They are required to fulfill certain criteria to select $W \rightarrow \tau \nu$ events [7]. After passing identification criteria the $\tau_{h}$ candidate is in addition required to have a single associated track to enrich the sample in true $\tau^{ \pm} \rightarrow$ $\pi^{ \pm} \pi^{0}$ decays. multijet background is suppressed by requiring large missing transverse energy, while contributions from other vector boson decays are reduced by rejecting events with additional light leptons. Remaining multijet background is estimated from control regions defined by varying the $\tau_{h}$ identification and $E_{T}^{\text {miss }}$ criteria. The electroweak background contributions are estimated from simulated samples. The energy of the charged pion is estimated from the track momentum associated to the $\tau_{h}$ candidate as measured by the inner detector, while the energy of the neutral pion is calculated as the difference of the energy deposition measured in the calorimeter and the track momentum. Figure 6 (a) shows the $p_{T}^{\text {trk }}$ spectrum for two samples reflecting the left- and right-handed $\tau$ hypotheses. Two distinct simulated $W \rightarrow \tau \nu$ signal samples were generated forcing the $\tau$ lepton to decay as left- or right-handed $\tau$ respectively. Figure 6 (b) shows the expected $\Upsilon$ spectrum from both samples along with the data. The polarization $P_{\tau}$ is extracted by a binned likelihood fit of the data to a parametrized template, obtained by linearly interpolating between the two simulated extreme cases of $P_{\tau}=1$ and $P_{\tau}=-1$ and its result is also shown in figure 6 (b). The 


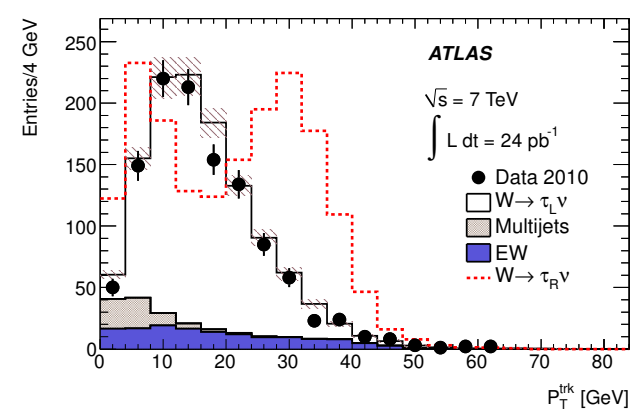

(a)

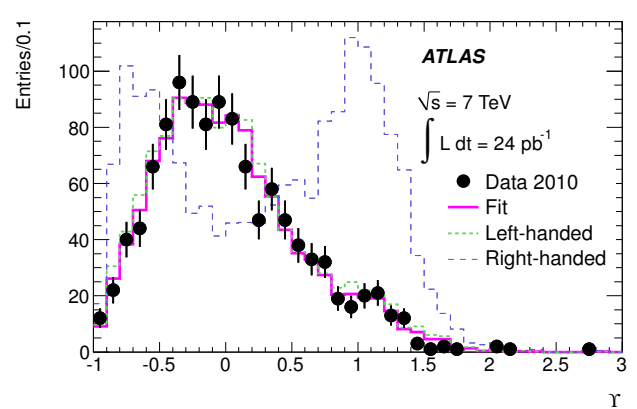

(b)

Figure 6: Distribution of $p_{T}^{\text {trk }}$ in data compared to the two simulated samples corresponding to $\tau_{\mathrm{L} / \mathrm{R}}$ (a) and the $\Upsilon$ distribution together with the extracted fit result. [7]

measured polarization is $P_{\tau}=-1.06 \pm 0.04$ (stat. $)_{-0.07}^{+0.05}$ (syst.) and well in agreement with the SM expectation.

\section{References}

[1] ATLAS Collaboration, The ATLAS Experiment at the CERN Large Hadron Collider, JINST 3 (2008) S08003.

[2] ATLAS Collaboration, Search for the Standard Model Higgs boson in the $H \rightarrow \tau^{+} \tau^{-}$decay mode in $\sqrt{s}=7 \mathrm{TeV}$ pp collisions with ATLAS, arXiv:1206.5971 [hep-ex].

[3] ATLAS Collaboration, Search for charged Higgs bosons decaying via $H^{ \pm} \rightarrow \tau v$ in top quark pair events using pp collision data at $\sqrt{s}=7 \mathrm{TeV}$ with the ATLAS detector, JHEP 1206 (2012) 039, arXiv:1204.2760 [hep-ex].

[4] ATLAS Collaboration, Search for supersymmetry with jets, missing transverse momentum and at least one hadronically decaying tau lepton in proton-proton collisions at $\sqrt{s}=7 \mathrm{TeV}$ with the ATLAS detector, arXiv:1204.3852 [hep-ex].

[5] ATLAS Collaboration, Search for events with large missing transverse momentum, jets, and at least two tau leptons in $7 \mathrm{TeV}$ proton-proton collision data with the ATLAS detector, arXiv: 1203.6580 [hep-ex].

[6] ATLAS Collaboration, Measurement of the top quark pair cross section with ATLAS in pp collisions at $\sqrt{s}=7 \mathrm{TeV}$ using final states with an electron or a muon and a hadronically decaying $\tau$ lepton, arXiv:1205.2067 [hep-ex].

[7] ATLAS Collaboration, Measurement of $\tau$ polarization in $W \rightarrow \tau \nu$ decays with the ATLAS detector in pp collisions at $\sqrt{s}=7 \mathrm{TeV}$, Eur. Phys. J. C (2012), arXiv:1204.6720 [hep-ex] .

[8] ATLAS Collaboration, Performance of the Reconstruction and Identification of Hadronic Tau Decays with ATLAS, Tech. Rep. ATLAS-CONF-2011-152, CERN. 